\title{
Exploring communication difficulties with deaf patients
}

\author{
Authors: Michel Abou-Abdallah ${ }^{A}$ and Abigail Lamyman ${ }^{B}$
}

One in five people in the UK are deaf, with hearing loss affecting more than $70 \%$ of people over the age of 70 . Despite this being a higher prevalence than asthma, heart disease or diabetes, deaf people experience persistent health inequalities with poorer experiences and outcomes in disease prevention and management. Clear communication and patient engagement with health are key to better outcomes. Nonetheless, there exist cultural, educational and organisational barriers when addressing communication with patients with hearing loss.

Foundation doctors have regular and prolonged contact with their patients, and often feel underprepared when interacting with patients with hearing loss. This article aims to highlight these communication barriers and suggest changes for improvement.

Improvement will require adaptations from both individual and organisational perspectives, with patient care as a clear focus for change.

KEYWORDS: foundation, communication, ENT, hearing, deaf

DOI: 10.7861/clinmed.2021-0111

\section{Introduction}

One in five people in the UK are deaf, rising to $70 \%$ in people over the age of 70, accounting for 12 million adults with hearing loss greater than 25 decibels hearing level (dB HL). A 2011 UK Census reported 22,000 residents reporting sign language as their main language, with the majority using British Sign Language (BSL). ${ }^{2}$ Among these patients are a group who identify as culturally Deaf, with a capital ' $D$ '. ${ }^{3}$ This cultural-linguistic minority experience the world from a unique perspective, which is poorly understood by many healthcare providers.

\section{Defining d/Deaf}

Profound hearing loss is defined as a hearing threshold of $90 \mathrm{~dB}$, at which level people will hear noises at least $50 \%$ of the time. ${ }^{4}$ It is

Authors: Afoundation year 2 doctor, Oxford University Hospitals NHS Trust, Oxford, UK; ${ }^{B}$ consultant ENT surgeon, Buckinghamshire Healthcare NHS Trust, Buckingham, UK important to note that conversational speech occurs at 50-60 dB HL, meaning that a 'moderate' hearing loss of up to $55 \mathrm{~dB}$ is functionally more significant than classified. Causes of hearing loss can be conductive, relating to abnormalities of the external ear or ossicles, sensorineural, relating to inner ear structures, or mixed. ${ }^{4} 70,000$ of the UK's deaf population are profoundly deaf prior to acquiring speech, ${ }^{5}$ and in developed countries $80 \%$ of congenital hearing loss is genetic. ${ }^{4}$ National Institute for Health and care Excellence guidance suggests the use of hearing aids for adults and children in whom hearing loss impairs their quality of life, and cochlear implantation in those without benefit after 3 months (unless contra-indicated or inappropriate). ${ }^{6}$ There exist socio-economic and health education barriers to hearing aid uptake, and people may choose not to be implanted for psychological and cultural reasons.?

Members of the Deaf community define deafness as a cultural identity rather than a disability. A report by Bonnie Poitras Tucker in 1998 describes the use of American Sign Language (ASL) as a visual language with its own syntax and grammar, quite different from spoken English. ${ }^{8}$ In the UK, the Deaf community is represented by organisations such as the British Deaf Association (BDA) and the Royal National Institute for Deaf People (RNID), who promote the positive aspects of being Deaf and lobby for changes such as the rights to communicate or be educated in their preferred language.

\section{Health inequalities}

Despite deafness having a higher prevalence than asthma, heart disease or diabetes, ${ }^{9}$ deaf people experience persistent health inequalities with poorer experiences and outcomes in disease prevention and management. ${ }^{3}$ Clear communication and patient engagement with health is key to better outcomes, which poses a challenge for the clinician unprepared to accommodate a patient's hearing needs.

Poor communication can lead to a lack of doctor-patient trust and to medical error. A survey by the RNID showed that $77 \%$ of BSL users had difficulty communicating with hospital staff, and 33\% left consultations with their family doctor unsure about instructions or taking incorrect medication doses. ${ }^{10} 30 \%$ avoided seeing their family doctors because of communication difficulties. $^{10}$

It is important to note that from August 2016, the Accessible Information Standard introduced a legal requirement in England for NHS organisations to ask for, record and meet communication needs. ${ }^{11}$ 


\section{Clinical case}

A recent trauma on-call shift saw the admission of a 95 -yearold male who had fallen and broken his hip. He was profoundly deaf from birth, with hearing aids in place bilaterally. Emergency department documentation had branded him a 'poor historian' and a largely collateral history was sought. Communication was through shouting and written information, which his daughter claimed was how he communicated at home. History taking, assessing cognition and surgical consent were challenging, but achieved through deliberated written notes. It became evident that these communication mediums were far from perfect. Fluctuating delirium and medical instability complicated the communicative effort, which raised a number of questions regarding his medical care:

> Was it clear to the medical team how much this patient understood and consented to?

> Would he have received different care had he been able to communicate verbally?

Sadly, this example is one we see all too often. Entries are still seen in patient records stating that a full history has not been taken because a patient is deaf ${ }^{5}$ and doctors interacting with these patients often feel ill-prepared to maximise the consultation and communicate effectively. ${ }^{12}$

This article aims to highlight the challenges faced by healthcare workers in communicating with patients with hearing loss. This aims to draw attention to the underlying factors leading to healthcare inequalities among the deaf community and to offer some guidance to approaching a consultation with patients with hearing loss.

\section{Barriers to communication}

\section{Lip reading}

Speaking loudly and slowly is technique often employed by the desperate doctor, with the aim of conveying information via lip reading and volume. Without sound, only $30 \%$ of English is readable on the lips, ${ }^{12}$ the rest being educated guessing. Knowing the topic in advance and any sound heard can improve the accuracy of these guesses. While we know that hearing loss in older age typically affects higher sounds first, ${ }^{12}$ using a deeper voice or increasing volume may distort the sound and prove less useful. Background noise should be kept to a minimum and the clinician should avoid obscuring their face and mouth and resist the urge to exaggerate words, which makes lip reading more difficult. $^{12}$

At present, these difficulties are compounded by the use of face masks for COVID-19 infection control. At present, these are opaque and obscuring of lip movement and visual facial cues, and have been demonstrated to impose additional barriers to hard of hearing patients $s^{13}$ and healthcare workers ${ }^{14}$ during the pandemic.

\section{Educational barriers}

In $2015,58.9 \%$ of deaf young people in England failed to achieve at least five GCSEs graded $A^{*}-C$, compared to $35.8 \%$ of people without special educational needs. They are also far less likely to go into higher education, and suffer a four-times higher unemployment rate. ${ }^{3}$ It may be convenient to assume that the acts of reading and writing do not involve the use of hearing, but this is a common misconception. Studies have found that reading comprehension scores in deaf students are significantly lower than in their hearing peers, ${ }^{15}$ a gap which is generally not made up in adult life. ${ }^{3}$ It is for these reasons which communication through a series of written notes does not replace the need for a carefully planned consultation.

Educational shortcomings in healthcare workers also contribute to the problem. Clinicians reportedly feel less comfortable with deaf patients, ${ }^{16}$ which may be rooted in a lack of education and understanding in communication tools. Anecdotally, BSL and communicating with deaf patients is a topic significantly underrepresented in medical school, which creates anxiety and poor performance when doctors are faced with this in practice.

\section{The use of interpreters}

An obvious solution is the use of medically trained interpreters in facilitating the consultation with a patient whose preferred language is BSL. As with interpreting for any language, using family or friends to interpret is suboptimal, with confidentiality concerns and the risk of information being translated incorrectly by accident or on purpose. ${ }^{3}$ Nonetheless, as many medical workers may have experienced, the practicality of achieving this is rarely as simple as the principle.

A study by Reeves in 2002 found that BSL interpreters were present at just $17 \%$ of GP and $7 \%$ of ED consultations, despite over half of the sample expressing desired support from these services. ${ }^{17}$ An interpreter had not been engaged for $42 \%$ of scheduled GP appointments where it would have been useful and $57 \%$ of A\&E attendees would have found it useful for the doctor or nurse to know BSL, or for there be a 24 -hour interpreter service. ${ }^{17}$ The 2018 Good Practice Report by the RNID echoes this, with $64 \%$ of patients leaving GP consultations feeling unclear at least some of the time. ${ }^{18}$

The reality is that while interpreting services are generally available, either in person or virtually, staff are often unaware of how to access them. Doctor-patient interactions in general practice or the hospital setting are often under time constraints outside the clinician's control and interpreters often need advance notice, with technological barriers to virtual consultations requiring specific apps and planning. ${ }^{17}$

\section{The confounding factor of cognitive state}

Hearing loss has been shown to be independently associated with delirium in the ED. ${ }^{19}$ Patients are concomitantly often affected by chronic cognitive conditions such as dementia. This must not be overlooked in the consultation, and specialised scoring systems such as the Hearing Impaired Montreal Cognitive Assessment (HIMOCA $)^{20}$ should be utilised when appropriate.

\section{Suggestions for change \\ Education for healthcare workers}

Deaf-awareness training is available to all healthcare workers and widely under-utilised. It may be beneficial for this training to become a compulsory module during medical school or foundation induction. It should be provided by members of the Deaf community, delivered in a thought-provoking and interactive way. ${ }^{5}$ Even basic courses in BSL may improve the confidence of 
doctors facing communication difficulties and raise awareness of the communication aids at their disposal.

Hearing aids can often be a source of anxiety amongst healthcare workers, with a lack of understanding of their function and maintenance. Ensuring hearing aids are functioning, switched on and being used appropriately by the patient is a small step towards successful communication.

Interpreter services should be signposted clearly at induction, with instructions on the necessary steps to access them. Often, video-interpreting requires preconfigured software on phones and tablets. Hospitals should prioritise making these available in key areas such the ED and easily accessible on the wards.

\section{Improving deaf representation}

It is important that we promote the engagement of deaf people in all aspects of healthcare. We should strive to make jobs in healthcare, health policy, research and education more accessible, maximising positive patient outcomes for deaf people. ${ }^{3}$ In doing this, we may see improvements in the socio-economic status of those who have hearing loss, and a greater representation of the Deaf community in clinical practice and health education. This will benefit not only the Deaf community, but their hearing colleagues.

\section{COVID-19 adjustments}

A number suggestions for improvements in communicating with the deaf or hard of hearing have been made. ${ }^{13,14}$ Clear face masks allow lip reading in face-to-face conversation and companies such as ClearMask ${ }^{\mathrm{M}}$ have begun supplying to the NHS, though there is a way to go before they are universally accessible. Captioning apps such as Google Live Transcribe can also help bridge the gap, allowing the speaker's voice to be converted live into text, with video conferencing programmes such as Google Meet and Microsoft Teams offering in-chat captioning. These work best in a quiet environment, and understanding must be double checked.

\section{Quick guide to communicating with patients with hearing loss}

Luckily, there are lots of resources to guide communication with patients with hearing loss. Taking inspiration from several sources ${ }^{12,21-23}$ I have compiled a quick 'AEIOU' guide for junior doctors finding themselves in this position (Box 1).

\section{Conclusion}

As foundation doctors, we have frequent and prolonged interactions with the patients under our care. We are often the clinician taking the admitting history, and a regular point of contact in clinic or on the ward. When done effectively, communication with patients who are deaf can improve their experiences and outcomes. Improving this requires adaptations from an individual and organisational perspective, with patient care as a clear focus for change.

\section{Acknowledgements}

I would like to thank my colleagues at Buckinghamshire Healthcare NHS Trust and Oxford University Hospitals NHS Foundation Trust for the experience, guidance and teaching which helped form this article.
Box 1. 'AEIOU' guide to communicating with patients with hearing loss ${ }^{12,21-23}$

Ask!

> How does the patient wish to communicate?

> Do their notes or GP records indicate their preferred language (eg British Sign Language)?

$>$ Do they have hearing aids, and are they working?

$>$ Is there a cognitive barrier that needs assessment?

\section{Environment}

$>$ Minimise background noise.

$>$ Ensure the interviewer's face is well lit and unobscured if possible.

> Dedicate time to the consultation; it may take longer than with hearing patients.

\section{Interaction}

> Speak clearly, avoiding exaggerated pronunciation.

> Use an interpreter when possible, making sure to address the patient not the interpreter.

\section{Outline}

> Prepare the patient for the topic of conversation.

$>$ Signpost changes in topic clearly.

\section{Understanding}

> Check patient comprehension and ask for periodic summaries. Repeat or rephrase unclear information.

\section{References}

1 Royal National Institute for Deaf People. Facts and figures. https:// rnid.org.uk/about-us/research-and-policy/facts-and-figures/.

2 Office for National Statistics. 2011 Census: Quick statistics for England and Wales, March 2011. ONS, 2013.

3 Rogers KD, Ferguson-Coleman E, Young A. Challenges of realising patient-centred outcomes for Deaf patients. Patient 2018;11:9-16.

4 Shearer AE, Hildebrand MS, Smith RJ. Hereditary hearing loss and deafness overview. In: GeneReviews ${ }^{\oplus}$, University of Washington, 2017.

5 Alexander A, Ladd P, Powell S. Deafness might damage your health. Lancet 2012;379:979-81.

6 National Institute for Health and Care Excellence. Cochlear implants for children and adults with severe to profound deafness. NICE, 2021.

7 Zhao F, Manchaiah V, Claire LS et al. Exploring the influence of culture on hearing help-seeking and hearing-aid uptake. Int J Audiol 2015;54:435-43.

8 Tucker BP. Deaf culture, cochlear implants, and elective disability. Hastings Cent Rep 1998;28:6-14.

9 Collins JG. Prevalence of selected chronic conditions: United States, 1990-1992. Vital Health Stat 10 1997;182:1-89.

10 Royal National Institute for the Deaf. A simple cure: a national report into deaf and hard of hearing people's experiences of the National Health Service. RNID, 2004.

11 NHS England. Accessible Information Standard. www.england.nhs. uk/ourwork/accessibleinfo/.

12 Barnett S. Communication with deaf and hard-of-hearing people: a guide for medical education. Acad Med 2002;77:694-700.

13 McKee M, Moran C, Zazove P. Overcoming additional barriers to care for deaf and hard of hearing patients during COVID-19. JAMA Otolaryngology Head Neck Surg 2020;146:781-2.

14 Grote H, Izagaren F. Covid-19: The communication needs of D/ deaf healthcare workers and patients are being forgotten. BMJ 2020;369:m2372. 
15 Traxler CB. The Stanford Achievement Test, 9th Edition: National norming and performance standards for deaf and hard-of-hearing students. J Deaf Stud Deaf Educ 2000;5:337-48.

16 Ralston E, Zazove P, Gorenflo DW. Physicians' attitudes and beliefs about deaf patients. J Am Board Fam Med 1996;9:167.

17 Reeves D, Kokoruwe B, Dobbins J, Newton V. Access to primary care and accident \& emergency services for Deaf people in the North West. NHS Executive North West Research and Development Directorate, 2002.

18 Royal National Institute for Deaf People. Good Practice. RNID, 2018. https://rnid.org.uk/wp-content/uploads/2020/07/Good practice-full-report.pdf [Accessed 28 March 2021].

19 Han JH, Zimmerman EE, Cutler $\mathrm{N}$ et al. Delirium in older emergency department patients: recognition, risk factors, and psychomotor subtypes. Acad Emerg Med 2009;16:193-200.
20 Lin VYW, Chung J, Callahan BL et al. Development of cognitive screening test for the severely hearing impaired: hearing-impaired MoCA. Laryngoscope 2017;127:S4-S11.

21 Fellinger J, Holzinger D, Pollard R. Mental health of deaf people. Lancet 2012;379:1037-44.

22 British Deaf Association. Communicating with deaf people. https:// bda.org.uk/help-resources/\#communicating.

23 Davidson M. Communicating with deaf patients. BMJ 2005;330:s 138

Address for correspondence: Dr Michel Abou-Abdallah, John Radcliffe Hospital, Headley Way, Headington, Oxford OX3 9DY, UK.

Email: Michel.abouabdallah@nhs.net 